\title{
GENERALIZATION OF LEVI-OKA'S THEOREM CONCERNING MEROMORPHIC FUNCTIONS
}

\author{
JOJI KAJIWARA and EIICHI SAKAI
}

Dedicated to Professor K. Noshiro on his sixtieth birthday

\section{Introduction}

As Fuks [3] stated, every domain of holomorphy or meromorphy over $C^{n}$ is analytically convex in the sense of Hartogs. Oka [6] proved that every domain over $C^{n}$ analytically convex in the sense of Hartogs is a domain of holomorphy. Therefore a domain of meromorphy over $C^{n}$ coincides with a domain of holomorphy over $C^{n}$.

In the present paper we shall prove that the envelope of meromorphy of a domain $(D, \varphi)$ over a Stein manifold $S$ with respect to a family of meromorphic functions on $D$ is $p_{i}$-convex in the sense of Docquier-Grauert [2] and, therefore, is a Stein manifold. Especially a domain of meromorphy over $S$ coincides with a domain of holomorphy over $S$.

A complex manifold $M$ is called of weak (or strong) Poincare type if for any meromorphic function $f$ on $M$ there exist holomorphic functions $g$ and $h$ on $M$ such that $f=g / h$ on $M$ (and that $g$ and $h$ are coprime at each point of $M$ ). From Siegel [8] any complex manifold of Cousin.II type is of strong Poincaré type and from Hitotumatu-Kôta [4] any Stein manifold is of weak Poincaré type.

Let $(D, \varphi)$ be a domain over a Stein manifold and $f$ be a meromorphic function on $D$. There exists a meromorphic function $\tilde{f}$ on the domain $\left(\tilde{\lambda}_{f}, \tilde{D}_{f}\right.$, $\left.\tilde{\varphi}_{f}\right)$ of meromorphy of $f$ such that $f=\tilde{f}_{0} \tilde{\lambda}_{f}$. As $\tilde{D}_{f}$ is a Stein manifold which is of weak Poincaré type, there exist holomorphic functions $\widetilde{g}$ and $\tilde{h}$ on $\tilde{D}_{f}$ such that $\tilde{f}=\tilde{g} / \tilde{h}$ on $\tilde{D}_{f}$. Then holomorphic functions $g=\tilde{g} \circ \tilde{\lambda}_{f}$ and $h=\tilde{h}_{h} \circ \tilde{\lambda}_{f}$ on $D$ satisfies $f=g / h$ on $D$. This means that any domain over a Stein manifold is of weak Poincaré type. 


\section{$\S 1$. Theorem of continuity}

Lемма 1. The following three assertions are valid for $n \geqq 2$.

1) If $f$ is meromorphic in a neighbourhood of $\bigcap_{p=1}^{\infty}\left\{z=\left(z_{1}, z_{2}, \ldots, z_{n}\right) ; z_{1}=a_{1}^{p}\right.$, $\left.z_{2}=a_{2}^{p}, \ldots, z_{n-1}=a_{n-1}^{p},\left|z_{n}\right| \leqq 1\right\} \cup\left\{z ; z_{1}=a_{1}^{0}, z_{2}=a_{2}^{0}, \ldots, z_{n-1}=a_{n-1}^{0},\left|z_{n}\right|=1\right\}$, $f$ can be meromorphically continued in a neighbourhood of. $\left\{z ; z_{1}=a_{1}^{0}, z_{2}=a_{2}^{0}\right.$, $\left.\ldots, z_{n-1}=a_{n-1}^{0},\left|z_{n}\right| \leqq 1\right\}$ where $a_{j}^{p} \rightarrow a_{j}^{0}$ as $p \rightarrow \infty$ for $j=1,2, \ldots, n-1$.

2) If $f$ is meromorphic in a neighbourhood of $\left\{z ;\left|z_{1}\right|=1, z_{2}=0, \ldots, z_{n-1}=0\right.$, $\left.0 \leqq z_{n} \leqq 1\right\} \cup\left\{z ;\left|z_{1}\right| \leqq 1, z_{2}=0, \ldots, z_{n-1}=0, z_{n}=0\right\}, f$ can be meromorphically continued in a neighbourhood of $\left\{z ;\left|z_{1}\right| \leqq 1, z_{2}=0, \ldots, z_{n-1}=0,0 \leqq z_{n} \leqq 1\right\}$.

3) If $f$ is meromorphic in a neighbourhood of $\left\{z ;\left|z_{1}\right|=1, z_{2}=0, \ldots, z_{n-1}=0\right.$, $\left.\left|z_{n}\right| \leqq 1\right\} \cup\left\{z ;\left|z_{1}\right| \leqq 1, z_{2}=0, \ldots, z_{n-1}=0, z_{n}=0\right\}, f$ can be meromorphically continued in a neighbourhood of $\left\{z ;\left|z_{1}\right| \leqq 1, z_{2}=0, \ldots, z_{n-1}=0,\left|z_{n}\right| \leqq 1\right\}$.

Proof. At first we shall prove the equivalence of 1), 2) and 3).

1) $\rightarrow 2$ ). Let $r$ be the supremum of $\delta>0$ such that $f$ can be meromorphically continued in a neighbourhood of

$$
C_{\delta}=\left\{z ;\left|z_{1}\right| \leqq 1, z_{2}=0, \ldots, z_{n-1}=0,0 \leqq z_{n} \leqq \delta\right\} .
$$

Suppose that $r \leqq 1$. Let $\left\{\delta^{p} ; p=1,2,3, \ldots\right\}$ be a sequence of positive numbers $\delta^{p}<\gamma$ such that $\delta^{p} \rightarrow \gamma$ as $p \rightarrow \infty$. Since $f$ is meromorphic in a neighbourhood of

$$
\begin{array}{r}
\bigcup_{p=1}^{\infty}\left\{z ;\left|z_{1}\right| \leqq 1, z_{2}=0, \ldots, z_{n-1}=0, z_{n}=\delta^{p}\right\} \cup\left\{z ;\left|z_{1}\right|=1, z_{2}=0, \ldots, z_{n-1}\right. \\
\left.=0, z_{n}=r\right\},
\end{array}
$$

$f$ can be meromorphically continued in a neighbourhood of $C_{r}$ from 1). Therefore we have $r \geqq 1$. Hence $f$ can be meromorphically continued in a neighbourhood of $C_{1}$.

$2) \rightarrow 3)$. Let $\theta$ be any real number. Since $f\left(z_{1}, z_{2}, \ldots, z_{n-1}, z_{n} \exp (\sqrt{-1} \theta)\right)$ is meromorphic in a neighbourhood of

$$
\left\{z ;\left|z_{1}\right|=1, z_{2}=0, \ldots, z_{n-1}=0,0 \leqq z_{n} \leqq 1\right\} \cup\left\{z ;\left|z_{1}\right| \leqq 1, z_{2}=0, \ldots, z_{n}=0\right\},
$$

$f\left(z_{1}, z_{2}, \ldots, z_{n-1}, z_{n} \exp (\sqrt{-1} \theta)\right)$ can be meromorphically continued in a neighbourhood of

$$
\left\{z ;\left|z_{1}\right| \leqq 1, z_{2}=0, \ldots, z_{n-1}=0,0 \leqq z_{n} \leqq 1\right\}
$$

from 2). Thus we have proved that $f\left(z_{1}, z_{2}, \ldots, z_{n}\right)$ can be meromorphically 
continued in a neighbourhood of

$$
\begin{aligned}
& \cup_{0 \leqq 0 \leqq 2 \pi}\left\{z ;\left|z_{1}\right| \leqq 1, z_{2}=0, \ldots, z_{n-1}=0,0 \leqq z_{n} \exp (-\sqrt{-1} \theta) \leqq 1\right\} \\
& \quad=\left\{z ;\left|z_{1}\right| \leqq 1, z_{2}=0, \ldots, z_{n-1}=0,\left|z_{n}\right| \leqq 1\right\} .
\end{aligned}
$$

$3) \rightarrow 1$ ). There exists $\delta>0$ such that $f$ is meromorphic in a neighbourhood of

$$
\left\{z ;\left|z_{1}-a_{1}^{n}\right|<2 \delta,\left|z_{2}-a_{2}^{0}\right|<2 \delta, \ldots,\left|z_{n-1}-a_{n-1}^{n}\right|<2 \delta,\left|z_{n}\right|=1\right\} .
$$

There exists $q>0$ such that $\left|a_{j}^{p}-a_{j}^{0}\right|<\delta(j=1,2, \ldots, n-1)$ for $p \geqq q$. Since $f$ is meromorphic in a neighbourhood of

$$
\begin{aligned}
\left\{z ; z_{1}=a_{1}^{q}, z_{2}=a_{2}^{q}, \ldots, z_{n-1}=a_{n-1}^{q},\left|z_{n}\right| \leqq 1\right\} \cup\left\{z ;\left|z_{1}-a_{1}^{q}\right| \leqq \delta,\right. \\
\left.z_{2}=a_{2}^{q}, \ldots, z_{n-1}=a_{n-1}^{q},\left|z_{n}\right|=1\right\},
\end{aligned}
$$

$f$ can be meromorphically continued in a neighbourhood of

$$
\left\{z ;\left|z_{1}-a_{1}^{q}\right| \leqq \delta, z_{2}=a_{2}^{q}, \ldots, z_{n-1}=a_{n-1}^{q},\left|z_{n}\right| \leqq 1\right\} .
$$

from 3). Of course $f$ can be meromorphically continued in a neighbourhood of

$$
\left\{z ; z_{1}=a_{1}^{0}, z_{2}=a_{2}^{q}, \ldots, z_{n-1}=a_{n-1}^{a},\left|z_{n}\right| \leqq 1\right\} .
$$

Continuing the same argument we can prove that $f$ can be meromorphically continued in a neighbourhood of

$$
\left\{z ; z_{1}=a_{1}^{0}, z_{2}=a_{2}^{0}, \ldots, z_{n-1}=a_{n-1}^{0},\left|z_{n}\right| \leqq 1\right\} .
$$

Okuda-Sakai [7] proved the validity of 1). Therefore 1),2) and 3) are all valid form the above discussion.

Lemma 2. If $f$ is meromorphic in $\left\{z=\left(z_{1}, z_{2}, \ldots, z_{n}\right) ; 1-\varepsilon<\left|z_{1}\right|<1+\varepsilon\right.$, $\left.\left|z_{2}\right|<1+\varepsilon, \ldots,\left|z_{n}\right|<1+\varepsilon\right\} \cup\left\{z ;\left|z_{1}\right| \leqq 1,\left|z_{2}\right|<1, \ldots,\left|z_{n}\right|<1\right\}$, f can be meromorphically continued in $\left\{z ;\left|z_{1}\right|<1+\varepsilon,\left|z_{2}\right|<1+\varepsilon, \ldots,\left|z_{n}\right|<1+\varepsilon\right\}$.

Proof. We take any $a_{j}$ with $\left|a_{j}\right|<1$ for $j=1,2, \ldots, n-1$. Let $\delta$ be any positive number with $\delta<1$. Since $f$ is meromorphic in a neighbourhood of

$$
\begin{array}{r}
\left\{z ;\left|z_{1}\right|=1, z_{2}=a_{2}, \ldots, z_{n-1}=a_{n-1},\left|z_{n}\right| \leqq 1+\varepsilon-\delta\right\} \cup\left\{z ;\left|z_{1}\right| \leqq 1, z_{2}=a_{2},\right. \\
\left.\ldots, z_{n-1}=a_{n-1}, z_{n}=0\right\},
\end{array}
$$

$f$ can be meromorphically continued in a neighbourhood of

$$
\left\{z ;\left|z_{1}\right| \leqq 1, z_{2}=a_{2}, \ldots, z_{n-1}=a_{n-1},\left|z_{n}\right| \leqq 1+\varepsilon-\delta\right\}
$$


from 3) of Lemma 1 . Therefore $f$ can be meromorphically continued in a neighbourhood of

$$
\left\{z ;\left|z_{1}\right| \leqq 1,\left|z_{2}\right|<1, \ldots,\left|z_{n-1}\right|<1,\left|z_{n}\right|<1+\varepsilon\right\} .
$$

Continuing the same argument we can prove that $f$ can be meromorphically continued in a neighbourhood of

$$
\left\{z ;\left|z_{1}\right| \leqq 1,\left|z_{2}\right|<1+\varepsilon, \ldots,\left|z_{n}\right|<1+\varepsilon\right\} .
$$

Therefore $f$ can be meromorphically continued in

$$
\left\{z ;\left|z_{1}\right|<1+\varepsilon,\left|z_{2}\right|<1+\varepsilon, \ldots,\left|z_{n}\right|<1+\varepsilon\right\} .
$$

\section{§ 2. Envelope of meromorphy}

In this section we shall define a meromorphic completion, an envelope of meromorphy and a domain of meromorphy. At the same time we can define a holomorphic completion, an envelope of holomorphy and a domain of holomorphy.

Let $M$ be a complex manifold. If there exists a local biholomorphic mapping of a complex manifold $V$ in $M,(V, \varphi)$ is called an open set over $M$. Moreover, if $V$ is connected, $(V, \varphi)$ is called a domain over $M$. Let $(V, \varphi)$ and $\left(V^{\prime}, \varphi^{\prime}\right)$ be open sets over $M$. If a holomorphic mapping $\lambda$ of $V$ in $V^{\prime}$ satisfies $\varphi=\varphi^{\prime} \circ \lambda, \lambda$ is called a mapping of $(V, \varphi)$ in $\left(V^{\prime}, \varphi^{\prime}\right)$. Consider domains $(V, \varphi)$ and $\left(V^{\prime}, \varphi^{\prime}\right)$ over $M$ with a mapping $\lambda$ of $(V, \varphi)$ in $\left(V^{\prime}, \varphi^{\prime}\right)$. Let $f$ be a meromorphic (or holomorphic) function on $V$. A meromorphic (or holomorphic) function $f^{\prime}$ on $V$ with $f=f^{\prime} \circ \lambda$ is called a meromorphic (or holomorphic) continuation of $f$ to $\left(\lambda, V^{\prime}, \varphi^{\prime}\right)$, or shortly to $\left(V^{\prime}, \varphi^{\prime}\right)$. Let $\approx$ be a family of meromorphic (or holomorphic) functions on $V$. If any meromorphic (or holomorphic) function of $\tilde{\xi}$ has a meromorphic (or holomorphic) continuation to $\left(\lambda, V^{\prime}, \varphi^{\prime}\right),\left(\lambda, V^{\prime}, \varphi^{\prime}\right)$, or shortly $\left(V^{\prime}, \varphi^{\prime}\right)$, is called a meromorphic (or holomorphic) completion of $(V, \varphi)$ with respect to the family $\tilde{\xi}$. A meromorphic (or holomorphic) completion $\left(\tilde{\lambda}_{\mathfrak{F}}, \tilde{V}_{\mathfrak{F}}, \tilde{\varphi}_{\mathfrak{F}}\right)$ of $(V, \varphi)$ with respect to $\tilde{F}$, or shortly $\left(\tilde{V}_{\mathscr{F}}, \tilde{\varphi}_{\mathscr{F}}\right)$, is called an envelope of meromorphy (or holomorphy) of $(V, \varphi)$ with respect to the family $\tilde{F}$ if the following conditions are satisfied:

Let $\left(\lambda^{\prime}, V^{\prime}, \varphi^{\prime}\right)$ be another meromorphic (or holomorphic) completion of $(V, \varphi)$ with respect to $\tilde{\xi}$. Then there exists a mapping $\psi$ of $\left(V^{\prime}, \varphi^{\prime}\right)$ in $\left(\widetilde{V}_{\mathfrak{F}}, \bar{\zeta}_{\mathfrak{F}}\right)^{\circ}$ 
with $\tilde{\lambda}_{\mathscr{F}}=\psi \circ \dot{\lambda}^{\prime}$ such that $\left(\psi, \tilde{V}_{\mathfrak{F}}, \tilde{\varphi}_{\mathfrak{F}}\right)$ is a meromorphic (or holomorphic) completion of $\left(V^{\prime}, \varphi^{\prime}\right)$ with respect to the family $\hat{\vartheta}^{\prime}$ of meromorphic (or holomorphic) continuations of all meromorphic (or holomorphic) functions of $\mathfrak{f}$.

If $\tilde{F}$ is the family of all meromorphic (or holomorphic) functions on $V$, a meromorphic (or holomorphic) completion of $(V, \varphi)$ with respect to $\tilde{F}$ and an envelope of meromorphy (or holomorphy) of $(V, \varphi)$ with respect to $\tilde{f}$ are called shortly a meromorphic (or holomorphic) completion of $(V, \varphi)$ and an envelope of meromorphy (or holomorphy) of $(V, \varphi)$ respectively.

Lemma 3. Let $(V, \varphi)$ be a domain over a complex manifold $M$ and $\left(\lambda, V^{\prime}\right.$, $\left.\varphi^{\prime}\right)$ be its meromorphic completion. Then $\left(\lambda, V^{\prime}, \varphi^{\prime}\right)$ is a holomorphic completion of $(V, \varphi)$.

Proof. Let $f$ be a holomorphic function on $V . \quad f$ has a meromorphic continuation $f^{\prime}$ to $\left(\lambda, V^{\prime}, \varphi^{\prime}\right)$. Since $\exp f$ must be meromorphically continued to the function $\exp f^{\prime}$ on $V^{\prime}, f^{\prime}$ must be holomorphic in $V^{\prime}$.

By the same method as Malgrange [5], who proved the unique existence of the envelope of holomorphy, we shall prove the unique existence of the envelope of meromorphy.

Lemma 4. Let $(V, \varphi)$ be a domain over a complex manifold $M$ and $\mathfrak{v}=\left\{f_{i}\right.$; $i \in I\}$ be a family of meromorphic functions on $V$. There exists uniquely an envelope of meromorphy of $(V, \varphi)$ with respect to $\mathfrak{F}$.

Proof. Consider an open neighbourhood $U$ of a point $a \in M$. Let $\left(g_{i}\right)_{i \in l}$ be a family of meromorphic functions in $U$ indexed by the above $I$. Let $\left(g_{i}^{\prime}\right)_{i \in l}$ be another such family defined in a neighbourhood $U^{\prime}$ of $a$. If there exists a neighbourhood $W$ of $a$ such that $W \subset U \cap U^{\prime}$ and $g_{i}=g_{i}^{\prime}$ in $W$ for any $i \in I$, $\left(g_{i}\right)_{i \in I}$ and $\left(g_{i}^{\prime}\right)_{i \in l}$ are identified. In this manner we shall induce an equivalence relation in the set of all families $\left(g_{i}\right)_{i \in I}$ of meromorphic functions defined in an open neighbourhood of $a$. An equivalence class is denoted by $\left(g_{i}\right)_{a}$ and the set of all classes $\left(g_{i}\right)_{a}$ is denoted by $\AA_{\mathfrak{F}, a}$. Let

$$
\Re_{\mathfrak{F}}=\bigcup_{\omega \in \mathbb{M}} \mathfrak{\Re}_{\mathfrak{F}, a} \text {. }
$$

We shall define a mapping $p$ of $\Re_{\mathfrak{F}}$ in $M$ by putting $p(x)=a$ for $x=\left(g_{i}\right)_{a} \in \Re_{\mathfrak{F}}$. We can induce on $\Re_{\mathfrak{F}}$ a sheaf structure as usual such that $\left(\AA_{\mathfrak{F}}, p\right)$ is an open set over $M$. If we define a mapping $\psi$ of $V$ in $\mathscr{R}_{\mathfrak{F}}$ by putting 


$$
\psi(a)=\left(f_{i} \circ \varphi^{-1}\right)_{\varphi(a)}
$$

for $a \in V, \psi$ is a mapping of $(V, \varphi)$ in $\left(\AA_{\mathfrak{F}}, p\right)$. The connected component of the complex manifold $\AA_{\mathfrak{F}}$ containing the connected open set $\psi(V)$ is denoted by $\widetilde{V}_{\mathfrak{F}}$. We put

$$
\tilde{\varphi}_{\mathfrak{F}}=p \mid \tilde{V}_{\mathfrak{F}}
$$

We shall define a meromorphic function $\widetilde{f}_{i}$ on $\tilde{V}_{\mathfrak{F}}$ for any $i \in I$ so as the germ defined by $\tilde{f}_{i}$ at $x=\left(g_{i}\right)_{a} \in \tilde{V}_{\mathfrak{F}}$, defined by a family of meromorphic functions $\left(g_{i}\right)_{i \in I}$ in a neighbourhood of $a$, coincides with the germ defined by $g_{i} \circ \tilde{\varphi}_{\mathfrak{F}}$ at $x$. For $x \in \psi(V)$, we have

$$
x=\left(f_{i} \circ \varphi^{-1}\right)_{\text {p }(a)}
$$

for $a \in V$. Therefore we have

$$
f_{i}=\tilde{f}_{i} \circ \psi
$$

for any $i \in I$. Hence $\tilde{f}_{i}$ is a meromorphic continuation of $f_{i}$ to $\left(\psi, \tilde{V}_{\mathscr{F}}, \tilde{\varphi}\right)$ for any $i \in I$. This means that $\left(\psi, \widetilde{V}_{\mathfrak{F}}, \tilde{\varphi}_{\mathfrak{F}}\right)$ is a meromorphic completion of $(V, \varphi)$ with respect to $\tilde{\mho}$.

Let $\left(\lambda, V^{\prime}, \varphi^{\prime}\right)$ be another meromorphic completion of $(V, \varphi)$ with respect to $\tilde{F}$. Let $f_{i}$ be any meromorphic function of $\mathfrak{F}$. There exists a meromorphic function $f_{i}^{\prime}$ on $V^{\prime}$ with $f_{i}=f_{i}^{\prime} \circ \lambda$. If we define a mapping $\psi^{\prime}$ of $V^{\prime}$ in $\Omega_{\mathfrak{F}}$ by putting

$$
\psi^{\prime}(a)=\left(f_{i}^{\prime} \circ \varphi^{1-1}\right)_{p^{\prime}(a)}
$$

for any $a \in V^{\prime}, \psi^{\prime}$ is a mapping of $\left(V^{\prime}, \varphi^{\prime}\right)$ in $\left(\Omega_{\mathfrak{F}}, p\right)$. Since $V^{\prime}$ is connected and $\psi(V) \subset \psi^{\prime}\left(V^{\prime}\right)$, we have

$$
\psi^{\prime}\left(V^{\prime}\right) \subset \widetilde{V} \mathfrak{F}
$$

Therefore $\psi^{\prime}$ is a mapping of $\left(V^{\prime}, \varphi^{\prime}\right)$ in $\left(\tilde{V}_{\mathscr{F}}, \tilde{\varphi}_{\mathscr{F}}\right)$ too. Moreover we have $\psi=\psi^{\prime} \circ \lambda$ and

$$
f_{i}^{\prime}=\tilde{f}_{i}^{\circ} \psi^{\prime}
$$

for any $i \in I$. Therefore $\left(\psi, \widetilde{V}_{\mathfrak{F}}, \tilde{\varphi}_{\mathfrak{F}}\right)$ is an envelope of meromorphy of $(V, \varphi)$ with respect to $\mathfrak{F}$.

Now let $(\tilde{\lambda}, \tilde{V}, \varphi)$ and $\left(\tilde{\lambda}^{\prime}, \tilde{V}^{\prime}, \tilde{\varphi}^{\prime}\right)$ be envelopes of meromorphy of $(V, \varphi)$ with respect to $\tilde{\xi}$. There exist, respectively, a mapping $\psi$ of $(\tilde{V}, \tilde{\varphi})$ in $\left(\tilde{V}^{\prime}, \tilde{\varphi}^{\prime}\right)$ and a mapping $\psi^{\prime}$ of $\left(\tilde{V}^{\prime}, \tilde{\varphi}^{\prime}\right)$ in $(\tilde{V}, \tilde{\varphi})$ such that 


$$
\lambda^{\prime}=\psi \circ \lambda, \lambda=\psi^{\prime} \circ \lambda^{\prime} .
$$

From the theorem of identity $\psi^{\prime} \circ \psi$ and $\psi^{\circ} \psi^{\prime}$ are, respectively, identities of $\widetilde{V}$ and $\tilde{V}^{\prime}$. Hence $\psi$ is biholomorphic. In this sense the envelope of meromorphy of $(V, \varphi)$ with respect to $\tilde{i}$ exists uniquely.

\section{§3. Pseudoconvexity of an envelope of meromorphy}

Lemma 5. Let $(V, \varphi)$ be a domain over an $n$-dimensional Stein manifold $S$, $\widetilde{V}=\left\{f_{i} ; i \equiv I\right\}$ be a family of meromorphic functions on $V$ and $\left(\tilde{\lambda}_{\mathfrak{F}}, \tilde{V}_{\mathfrak{F}}, \tilde{\varphi}_{\mathfrak{F}}\right)$ be the envelope of meromorphy of $(V, \varphi)$ with respect to $\mathfrak{F}$. Then $\left(\tilde{V}_{\mathfrak{F}}, \tilde{\varphi}_{\mathfrak{F}}\right)$ is $p_{i-}$ convex in the sense of Docquier-Grauert [2] and, therefore, $\tilde{V}_{\Im}$ is a Stein manifold.

Proof. We may suppose that $n \geqq 2$. We put

$$
D=\left\{z=\left(z_{1}, z_{2}, \ldots, z_{n}\right) ;\left|z_{1}\right| \leqq 1,\left|z_{2}\right|<1, \ldots,\left|z_{n}\right|<1\right\}
$$

and

$$
\delta D=\left\{z ;\left|z_{1}\right|=1, z \in D\right\} .
$$

Consider a continuous mapping $\psi$ of the closure $\bar{D}$ of $D$ in $\widetilde{V}_{\Im} \cup \widetilde{\partial} \widetilde{V}_{\mathfrak{F}}$ with the following properties :

1) $\varphi(\delta D) \Subset \tilde{V}_{\Im}$

2) $\psi(\grave{D}) \subset \widetilde{V}_{\mathfrak{F}}$

3) $\tilde{\varphi}_{F^{\circ}} \circ$ can be continued to a biholomorphic mapping $\xi$ of a neighbourhood of $\bar{D}$ in $S$.

From 3) $\xi$ is a biholomorphic mapping of $B_{\S}$ in $S$ for $0<\varepsilon \leqq \varepsilon^{\prime}$ where

$$
B_{\varepsilon}=\left\{z ;\left|z_{1}\right|<1+\varepsilon,\left|z_{2}\right|<1+\varepsilon, \ldots,\left|z_{n}\right|<1+\varepsilon\right\}
$$

and $\varepsilon^{\prime}$ is a suitable positive number. If we put $G_{\varepsilon}=\xi\left(B_{\varepsilon}\right), \tilde{\varphi}_{\mathfrak{F}} \operatorname{maps} \tilde{\varphi}_{\mathfrak{F}}^{-1}\left(G_{\varepsilon}\right)$ biholomorphically on the subdomain $G_{\varepsilon}$ of $S$ for $0<\varepsilon \leqq \varepsilon^{\prime}$. From 1) and 2) $\psi$ can be regarded as a biholomorphic mapping of $C_{\varepsilon}$ in $\tilde{V}_{\mathscr{F}}$ for $0<\varepsilon \leqq \varepsilon^{\prime \prime}$. where

$$
C_{\varepsilon}=D \cup\left\{z ; 1-\varepsilon<\left|z_{1}\right|<1+\varepsilon,\left|z_{2}\right|<1+\varepsilon, \ldots\left|z_{n}\right|<1+\varepsilon\right\}
$$

and $\varepsilon^{\prime \prime}$ is a suitable positive number.

Now let $f_{i}$ be any meromorphic function of $\tilde{F}$ and $\tilde{f}_{i}$ be its meromorphic continuation to $\left(\tilde{\lambda}_{\mathscr{F}}, \tilde{V}_{\mathfrak{F}}, \tilde{\varphi}_{\mathscr{F}}\right)$. Then $g_{i}=\tilde{f}_{i}^{\circ} \phi$ is meromorphic not only in $D$ but also in $C_{\varepsilon}$ for $0<\varepsilon \leqq \delta=\min \left(\varepsilon^{\prime}, \varepsilon^{\prime \prime}\right)$. Lemma 4 means that $B_{\varepsilon}$ is a meromorphic completion of $C_{\varepsilon}$. Therefore there exists a meromorphic contination $\widetilde{g}_{i}$ of $g_{i}$ 
to $B_{\hat{r}}$. We shall consider the sum space $\widetilde{V}_{\mathfrak{F}} \cup B_{\delta}$. We shall identify a point $x \in \tilde{V}_{\mathfrak{F}}$ and a point $y \in B_{\delta}$ if

$$
\tilde{\varphi}_{\mathfrak{F}}(x)=\xi(y),\left(\tilde{f}_{i} \circ \tilde{\varphi}_{\mathfrak{F}^{-1}}^{-1}\right)_{\mathcal{F}_{\mathfrak{F}}(x)}=\left(\widetilde{g}_{i} \circ \xi^{-1}\right)_{\xi(y)} .
$$

We can put a complex structure on the quotient space $V^{\prime}$ of $\widetilde{V}_{\mathfrak{F}} \cup B_{\delta}$ by the equivalence relation induced by the above identification. The holomorphic mappings $\varphi$ and $\xi$ induce naturally a local biholomorphic mapping $\varphi^{\prime}$ of $V^{\prime}$ in $S$. The natural injection $\widetilde{V}_{\mathfrak{F}} \rightarrow \widetilde{V}_{\mathfrak{F}} \cup B_{\delta}$ induces a biholomorphic mapping $i$ of $\tilde{V}_{\mathfrak{F}}$ in $V^{\prime} . \quad i$ is a mapping of $\left(\tilde{V}_{\mathfrak{F}}, \tilde{\varphi}_{\mathfrak{F}}\right)$ in $\left(V^{\prime}, \varphi^{\prime}\right)$. Since $\left(i \circ \tilde{\lambda}_{\mathfrak{F}}, V^{\prime}, \varphi^{\prime}\right)$ is a meromorphic completion of $(V, \varphi)$ with respect to $\tilde{F}$ and since $\left(\tilde{\lambda}_{\mathfrak{F}}, \tilde{V}_{\mathfrak{F}}, \tilde{\varphi}_{\mathfrak{F}}\right)$ is the envelope of meromorphy of $(V, \varphi)$ with respect to $\tilde{\xi}$, there exists a mapping $j$ of $\left(V^{\prime}, \varphi^{\prime}\right)$ in $\left(\tilde{V}_{\mathfrak{F}}, \tilde{\varphi}_{\mathfrak{F}}\right)$ such that $\tilde{\lambda}_{\mathfrak{F}}=j \circ i \circ \tilde{\lambda}_{\mathfrak{F}}$. From the theorem of identity $j \circ i$ is the identity of $\widetilde{V}_{\mathfrak{F}}$. The natural injection $B_{\delta} \rightarrow V_{\Im} \cup B_{\delta}$ induces a biholomorphic mapping $\psi^{\prime}$ of $B_{\delta}$ in $V^{\prime}$. It holds that

$$
\psi^{\prime}=i \circ \psi
$$

in $D \Subset B_{\delta}$. Therefore we have

$$
\psi=j \circ \psi^{\prime}
$$

in $D$. Hence we have

$$
\psi(D) \Subset V_{\mathfrak{F}}
$$

as $\psi^{\prime}(D) \Subset V^{\prime}$. Thus we have proved that $\left(\widetilde{V}_{\mathfrak{F}}, \tilde{\varphi}_{\mathfrak{F}}\right)$ is $p_{7}$-convex in the sense of Docquier-Grauert [2]. Of course $\widetilde{V}_{\mathfrak{F}}$ is a Stein manifold from [2].

\section{§. Main results}

Let $(V, \varphi)$ be a domain over a complex manifold $M$ and $f$ be a meromorphic (or holomorphic) function on $V$. The envelope $\left(\tilde{\lambda}_{f}, \tilde{V}_{f}, \tilde{\varphi}_{f}\right)$ of meromorphy (or holomorphy) of $(V, \varphi)$ with respect to the family consisting of only $f$ is called the domain of meromorphy (or holomorphy) of $f$. A domain over $M$ is called a domain of meromorphy (or holomorphy) if it is a domain of meromorphy of a meromorphic (or holomorphic) function on a domain over $M$.

Proposition 1. Let $(V, \varphi)$ be a domain over a Stein manifold $S$. Then $V$ is of weak Poincare type.

Proof. Let $f$ be a meromorphic function on $V$ and $\left(\tilde{\lambda}_{f}, \tilde{V}_{f}, \tilde{\varphi}_{f}\right)$ be the domain of meromorphy of $f$. There exists a meromorphic continuation $\tilde{f}$ of $f$ 
to $\left(\tilde{\lambda}_{f}, \widetilde{V}_{f}, \tilde{c}_{f}\right)$. From Lemma $5 \tilde{V}_{f}$ is a Stein manifold, which is of weak Poincaré type from Hitotumatu-Kôta [3]. There exist holomorphic functions $\widetilde{g}$ and $\tilde{h}$ on $\tilde{V}_{f}$ such that $\tilde{f}=\widetilde{g} / \widetilde{h}$ on $\tilde{V}_{\mathscr{\mho}}$. If we put $g=\widetilde{g}^{\circ} \tilde{\lambda}_{f}$ and $h=\tilde{h}_{\circ} \circ \tilde{\lambda}_{f}, g$ and $h$ are holomorphic functions on $V$ such that $f=g / h$ on $V$.

If we put

$$
S=C^{n} \times P(n \geqq 1),
$$

$S$ is a non compact holomorphically convex complex manifold which is not of weak Poincaré type. The authors do not know whether there exists a holomorphically convex complex manifold which is obtained by a proper modification of a Stein space and a domain over which is not of weak Poincaré type. We shall prove the converse of Lemma 3 for domains over a Stein manifold.

Proposition 2. Let $(V, \varphi)$ be a domain over a Stein manifold $S$ and $\left(\lambda, V^{\prime}, \varphi^{\prime}\right)$ be its holomorphic completion. Then $\left(\lambda, V^{\prime}, \varphi^{\prime}\right)$ is a meromorphic completion of $(V, \varphi)$.

Proof. Let $f$ be a meromorphic function on $V$. From Proposition 1 there exist holomorphic functions $g$ and $h$ on $V$ such that $f=g / h$ on $V$. There exist holomorphic continuations $g^{\prime}$ and $h^{\prime}$ of $g$ and $h$ to $\left(\lambda, V^{\prime}, \varphi^{\prime}\right)$ respectively. Then $f^{\prime}=g^{\prime} / h^{\prime}$ is a meromorphic continuation of $f$ to $\left(\lambda, V^{\prime}, \varphi^{\prime}\right)$.

Corollary. Let $(V, \varphi)$ be a domain over a Stein manifold. Then $\left(\lambda, V^{\prime} . \varphi^{\prime}\right)$ is a holomorphic completion of $(V, \varphi)$ if and only if it is a meromorphic completion of $(V, \varphi)$.

As a special case of the above Corollary we have the following Proposition.

Proposition 3 Let $(V, \varphi)$ be a domain over a Stein manifold $S$. Then the envelope $\left(\tilde{\lambda}_{\Re}, \bar{V}_{\Re}, \tilde{\varphi}_{\Re}\right)$ of meromorphy of $(V, \varphi)$ coincides with the envelope ( $\tilde{\lambda}_{\mathscr{O}}$, $\left.V_{D}, \tilde{\varphi}_{D}\right)$ of holomorphy of $(V, \varphi)$.

Proof. From Lemma 3 and Proposition 2 there exist, respectively, a map-

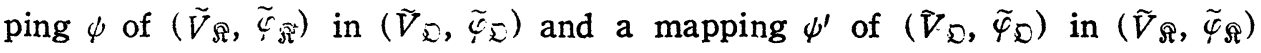
such that $\lambda=\psi^{\circ} \lambda^{\prime}$ and $\lambda^{\prime}=\psi^{\prime} \circ \lambda$. From the theorem of identity $\psi^{\prime} \circ \psi$ and $\psi^{\circ} \psi^{\prime}$ are, respectively, identities of $\tilde{V}_{\Re}$ and $\tilde{V}_{\mathcal{O}}$. Hence $\psi$ is a biholomorphic mapping of $\tilde{V}_{\Re}$ in $V_{\Omega}$. In this sense $\left(\tilde{\lambda}_{\Re}, \tilde{V}_{\Re}, \tilde{\varphi}_{\Re}\right)$ coincides with $\left(\tilde{\lambda}_{D}, \tilde{V}_{\mathcal{O}}, \tilde{\varphi}_{\mathscr{D}}\right)$.

Theorem. Let $(V, \varphi)$ be a domain over a Stein manifold $S$. Then the following 
assertions are equivalent:

1) $(V, \varphi)$ is an envelope of meromorphy with respect to a family of meromorphic functions on a domain over $S$.

2) $(V, \varphi)$ is a domain of meromorphy.

3) $(V, \varphi)$ is domain of holomorphy.

4) $V$ is holomorphically convex.

Proof. From Lemma 5 1) implies 4). Ouite similarly as in the proof of Lemma 5,4$)$ follows from 3) by Docquier-Grauert [2]. If $V$ is holomorphically convex, $(V, \varphi)$ is a domain of meromorphy of a holomorphic function on $V$ from Cartan-Thullen [1]. A domain of meromorphy of a meromorphic function is an envelope of meromorphy with respect to the family consisting of only $f$.

Roughly speaking, the theory of domains of meromorphy over a Stein manifold coincides almost with the theory of domains of holomorphy over $C^{n}$.

\section{REFERENCES}

[1] H. Cartan und P. Thullen, Zur Theorie der Singularitäten den Funktionen mehrerer komplexen Veränderlichen: Regularitäts -und Konvergenz-bereiche, Math. Ann., 106 (1932), 617-647.

[2] F. Docquier und H. Grauert, Levisches Problem und Rungescher Satz für Teilgebiete Steinscher Mannigfaltigkeit, Math. Ann., 140 (1960), 94-123.

[3] B. A. Fuks, Special chapters of theory of analytic functions of several complex variables, Moscow (1963).

[4] S. Hitotumatu and O. Kôta, Ideals of meromorphic functions of several complex variables, Math. Ann., 125 (1952), 119-126.

[5] B. Malgrange, Lectures on the theory of functions of several complex variables, Tata Inst. Fund. Res. Bombay, (1958).

[6] K. Oka, Sur les fonctions analytiques de plusieur variables IX: Domaines finis sans point critique intérieur, Jap. J. Math., 27 (1953), 67-155.

[7] H. Okuda and E. Sakai, On the continuation theorem of Levi and the radius of meromorphy, Mem. Fac. Sci. Kyushu Univ. (A), 11 (1957), 65-73.

[8] C. L. Siegel, Analytic functions of several complex variables, Priceton Lecture (1949/50).

\section{Mathematical Institute, Nagoya University}

and

Mathematical Institute, Kanazawa University 